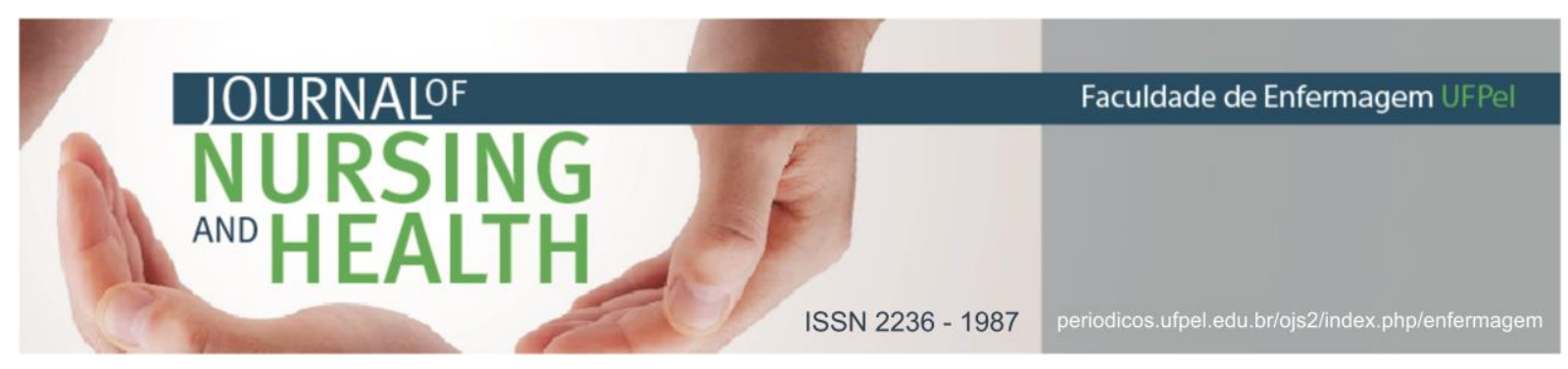

RELATO DE EXPERIÊNCIA

\title{
A realização de intercâmbio no doutorado em enfermagem: um relato de experiência*
}

\author{
The realization of exchange in the doctorate in nursing: an experience report \\ La realización de intercambio en el doctorado en enfermería: un relato de \\ experiencia
}

Bardaquim, Vanessa Augusto1; Dias, Ernandes Gonçalves²

Como citar este artigo: Bardaquim VA, Dias EG. A realização de intercâmbio no doutorado em enfermagem: um relato de experiência. J. nurs. health. 2019;9(1):e199104

\section{RESUMO}

Objetivo: relatar a experiência discente de pós-graduação de Enfermagem em Programa de intercâmbio internacional. Método: trata-se de um relato de experiência, sobre a participação no programa de Doutorado Sanduíche, realizado por meio de concessão de bolsa do Programa de Coordenação de Aperfeiçoamento de Pessoal de Nível Superior, entre a Escola de Enfermagem de Ribeirão Preto, São Paulo e a Universidade Laval, Quebec, entre agosto a dezembro de 2017. Resultados: participação em cursos, palestras e eventos, aluna ouvinte em disciplinas relacionadas à prática da enfermagem. Estratégia de aprendizagem baseada em problemas, as atividades de ensino foram realizadas em forma de apresentações audiovisuais, discussões em sala de aula, atividades em laboratório, trabalho em equipe, material multimídia, videoconferências, tutoriais e plataforma web. Considerações Finais: o intercambio é oportunidade para agregar valores, aprimorar o currículo e ampliar o conhecimento, além de conhecer diferentes linhas de pesquisas e teorias voltadas para a área da saúde.

Descritores: Intercâmbio educacional internacional; Enfermagem transcultural; Enfermagem.

\begin{abstract}
Objective: to report the graduate student experience of Nursing in International Exchange Program. Method: this is an experience report about the participation in the Sandwich Doctoral Program, carried out by means of a grant from the Coordination Program for the Improvement of Higher Education Personnel, between the School of Nursing of Ribeirão Preto, São Paulo and Laval University, Quebec, from August to December 2017. Results: participation in courses, lectures and events, student listener in disciplines related to nursing practice. Problem-based learning strategy, teaching activities were conducted in the form of audiovisual presentations, classroom discussions, laboratory activities, teamwork, multimedia material, video conferences, tutorials and web platform. Final Considerations: the exchange is an opportunity to aggregate values, to improve the
\end{abstract}

*O presente trabalho foi realizado com apoio da Coordenação de Aperfeiçoamento de Pessoal de Nível Superior (CAPES) - Brasil. Código de Financiamento 001.

1 Enfermeira. Mestre em Enfermagem. Universidade de São Paulo (USP). E-mail: va.bardaquim@bol.com.br http://orcid.org/0000-0003-2179-552X

2 Enfermeiro. Mestre em Ciências. Faculdade Verde Norte (FAVENORTE). E-mail: ernandesgdias@yahoo.com.br http: / /orcid.org/0000-0003-4126-9383 


\section{JOURNALOF

curriculum and to increase knowledge, besides knowing different lines of research and theories focused on the health area.

Descriptors: International educational exchange; Transcultural nursing; Nursing

\section{RESUMEN}

Objetivo: informe sobre la experiencia discente de postgrado de Enfermería en Programa de intercambio internacional. Método: se trata de un relato sobre la participación en el Doctorado Sándwich, realizado por medio de concesión de beca del Programa de Coordinación de Perfeccionamiento de Personal de Nivel Superior, entre la Escuela de Enfermería de Ribeirão Preto, São Paulo y la Universidad Laval, Quebec, entre agosto y diciembre de 2017. Resultados: participación en cursos, charlas y eventos, alumna oyente en disciplinas relacionadas a la práctica. La estrategia de aprendizaje basada en problemas, las actividades de enseñanza se realizaron en forma de presentaciones audiovisuales, discusiones en el aula, actividades en laboratorio, trabajo en equipo, material multimedia, videoconferencias, tutoriales y plataforma web. Consideraciones finales: el intercambio es una oportunidad para agregar valores, mejorar el currículo y ampliar el conocimiento, además de conocer diferentes líneas de investigación y teorías orientadas al área de la salud.

Descriptores: Intercambio educacional internacional; Enfermería transcultural; Enfermería.

\section{INTRODUÇÃO}

A globalização da educação superior é considerada um conceito complexo e interconectado, com termos, como mundialização, internacionalização da educação, cooperação internacional, os quais passam por interferências no tempo e no desenvolvimento de relações internacionais para a educação a nível superior. ${ }^{1-2}$

Dessa forma, o intercâmbio proporciona aos pesquisadores aprimorar o conhecimento científico e teórico, através das trocas de experiências, de pesquisas, a nível cultural, da situação política, econômica e ações desenvolvidas entre as universidades e os serviços de saúde. $^{3-4}$ Essas parcerias entre as instituições estrangeiras devem ser vivenciadas por discentes que almejam um diferencial na profissão e que buscam a internacionalização, isto é, desenvolver suas habilidades e competências profissionais no campo da academia internacional. ${ }^{2}$
No Brasil é crescente o interesse e a procura de estudantes tanto da graduação como da pós-graduação nas mais variadas áreas pela internacionalização. Em relação a área da enfermagem, o intercâmbio contribui para a aproximação dos estudantes às novas realidades práticas e de saberes, o que colabora para a formação e um melhor desempenho profissional. ${ }^{5}$

Contudo, historicamente o Canadá vem se destacando na sociedade e na construção de competências para uma saúde de qualidade, ${ }^{6}$ atraindo jovens estudantes de várias partes do Mundo. ${ }^{7-9}$

Assim, este relato de experiência teve como objetivo relatar a experiência discente de pós-graduação de Enfermagem em um Programa de intercâmbio internacional. Como também, ser meio de incentivo e apoio aos interessados a realizarem 0 intercâmbio acadêmico. ${ }^{3}$ 


\section{MÉTODOS}

Relato de experiência sobre o estágio intitulado Doutorado Sanduíche realizado por meio de concessão de bolsa do Programa de Coordenação de Aperfeiçoamento de Pessoal de Nível Superior (CAPES) - Brasil, entre a Escola de Enfermagem de Ribeirão Preto da Universidade de São Paulo (USP) e a Université Laval (Quebec/Canadá).

Foram desenvolvidas atividades na Universidade e no laboratório de pesquisa no período de agosto a dezembro de 2017, junto aos Programas de Pós-Graduação entre as Universidades do curso de Enfermagem, cujo campo de estudo foi as condições crônicas não transmissíveis e a discussão do projeto de pesquisa sobre o estresse na equipe de enfermagem da área hospitalar.

0 processo seletivo foi guiado pelo Edital da CAPES, 19/2016 e organizado pela coordenação do Programa de Pós-Graduação que compôs uma Comissão para a seleção dos candidatos, composta por três membros: o Coordenador do Programa, um representante discente doutorando e um avaliador externo ao Programa.

Para cursar o doutorado sanduíche no exterior, os pretendentes a vaga deveriam preencher os seguintes pré-requisitos: ser aluno regularmente matriculado em cursos de doutorado no Brasil e se propor realizar parte do curso em instituição no exterior em um período de no mínimo de três e no máximo 12 meses, com retorno ao Brasil para a integralização de créditos e defesa da tese.
Desta forma, participaram da seleção alunos do curso de doutoramento, com a finalização deste processo e aprovação em março de 2017. Após a confirmação dos resultados e da obtenção da bolsa de estudos, iniciaram-se os trâmites para documentação, o deslocamento e a instalação.

\section{RESULTADOS E DISCUSSÃO}

\section{Atividades desenvolvidas no Doutorado Sanduíche}

Os estudos foram realizados no hospital denominado Centre de Recherche Institut Universitaire de Cardiologie et de Pneumologie de Quebec, onde possui laboratórios de pesquisas e na Université Laval, onde havia uma sala de estudos exclusiva e destinada para estudantes de pósgraduação.

A aprendizagem durante o estágio Sanduíche foi dinâmica, de acordo com o cronograma realizava-se atividades diversificadas, entre cursos de idiomas em francês, diariamente pela manhã, e no período da tarde realizava-se atividades na Université Laval e/ou no laboratório de pesquisa inserido no hospital. Dessa maneira optou-se por categorizar as experiências em temas para facilitar a compreensão do leitor.

\section{Atividades de ensino e aprendizagem no intercâmbio}

Considerando que a língua oficial do campo de estágio é o Francês tevese a necessidade de realizar aulas do idioma, assim foi realizado 94 horas/aula no programa de integração de Francização, na Escola Centre 


\section{NURSING \\ ANO HEALTH}

MgrMarcoux

no

Centre

Communautaire et de Loisir.

Ao chegar à Universidade em agosto de 2017, deparou-se com o período de férias, porém, identificouse que havia outros tipos de cursos de Verão (École d'été) na Faculdade de Enfermagem (Facultéd essciences Infirmières), sendo possível participar de palestras diariamente, por quinze dias.

O calendário letivo iniciou no mês de setembro de 2017, quando foi oportunizado participar como aluna ouvinte em duas disciplinas. A "Prática de Enfermagem" (Pratique Infirmières), destinada a ministrar aulas na graduação para prática de enfermagem no contexto da área hospitalar. Esta disciplina reportava-se aos pacientes vulneráveis, em estado crítico de saúde e suas orientações aos familiares. Os cuidados de enfermagem eram contextualizados de acordo como modelo teórico da autora Virginia Henderson.

A teoria de Henderson tem sido empregada em estudos internacionais, considerada uma teoria simples e autoexplicativa que pode ser utilizada em diferentes contextos, envolvendo as necessidades humanas e o cuidar em enfermagem. ${ }^{10}$

Em seguida, participou-se também como aluna ouvinte na disciplina "Fundamentos Biomédicos" (Fondements Biomédicales), complementar à disciplina Práticas de enfermagem (Pratique Infirmières), com abordagem na fisiopatologia das condições clínicas mais frequentes encontradas em pacientes no contexto de cuidados críticos. Empregou-se
ISSN 2236 - 1987

estratégia de aprendizagem baseada em problemas.

Considera-se a problematização uma estratégia eficaz de ensinoaprendizagem, que confere autonomia ao estudante para a preservação da integralidade do ser humano. ${ }^{11} \mathrm{~A}$ disciplina também abordava os cuidados de enfermagem ao paciente no contexto das doenças cardíacas e pacientes em estado crítico. A assistência do enfermeiro aos portadores de cardiopatia é considerada importante, pois atua na promoção da saúde, atendimento humanizado, exerce a função de educador e realiza planejamento da capacitação da equipe de enfermagem. ${ }^{12}$

Os métodos de aprendizado utilizado pelos professores e técnicos de laboratório na Universidade eram os mais diversificados. As atividades de ensino foram realizadas em forma de apresentações audiovisuais, estudos de casos clínicos, discussões em sala de aula, atividades em laboratório sobre as técnicas e cuidados de enfermagem, trabalho em equipe, participações de palestrantes especialistas, material multimídia, tutoriais e plataforma web.

\section{Participações em eventos}

Foi possível ainda participar de palestras, como ouvinte, voltadas para a área da saúde, com temas atuais e relevantes para a área hospitalar. Os temas foram: "Le don d'organeencontexte d'aidemédicale à mourir" (a doação de órgãos no contexto de morte assistida), "La priseen charge desbrulésaux soins intensifs" (cuidados aos pacientes 
queimados na Unidade de Terapia Intensiva (UTI)), "Les incontournables enchirurgie vasculaire" (elementos essenciais em cirurgia vascular), "Les soins intensifssoussilence" (Os ruídos nas UTI), "Promotion de la certification" (promoção da certificação da enfermeira assistentechefe), "Déséquilibre hydroélectrolytique aux soins intensifs" (desequilíbrio hidroeletrolítico em UTI), “Le leadership d'une infirmière/où ça peut nous mener" (a liderança em enfermagem), "Premier Café-conférence" (A primeira conferência do café) com apresentação de resultados parciais de pesquisas e estudos clínicos randomizados.

Participação, como aluna ouvinte, em videoconferências, cujos temas eram: Hipertensão Arterial Sistêmica, equino-oxigenação transmembrana, promoção da saúde, comportamentos de saúde, teorias para propor intervenções de enfermagem na prática clínica. Ao mesmo tempo, teve reunião com a bibliotecária para buscar artigos científicos, nas principais bases de dados relevantes para área da saúde.

Ocasionalmente, realizou-se auxílio nas salas de aulas na aplicação de provas para os alunos de Graduação em Enfermagem e semanalmente reunião com a orientadora supervisora do estágio sanduíche sobre as atividades realizadas e apresentação dos resultados e discussão do projeto de pesquisa do doutorado.

Nesse ínterim, foi possível participar da $10^{\mathrm{a}}$ edição do evento anual "Soirée reconnaissance", em que a direção da Faculdade de Enfermagem destaca e homenageiam alunos da graduação e da pós-graduação, docentes e funcionários pelo dossiê acadêmico para a obtenção de bolsas, subvenções de prestígio e prêmios, pelo trabalho de supervisão de estágio ou realizações e projetos de pesquisa.

Houve participação como aluna ouvinte em videoconferência em banca de doutorado entre Brasil e Canadá. Ainda, participação no evento "Journée de Formation", destinado aos cuidados de enfermagem em unidades de terapia intensiva ("Soins Intensifs"), bem como em reuniões com os alunos que estão desenvolvendo projetos piloto em pesquisas quantitativas e sobre 0 Comitê de Ética em Pesquisa Canadense.

\section{Desafios e potencialidades vivenciadas}

Observa-se no Canadá o respeito que os governantes têm com a sociedade de uma forma geral, toda a infraestrutura, a organização, as taxas de impostos, o transporte público, o conforto dos ambientes e a educação. Contudo, surgem os desafios e as dificuldades no intercâmbio, que foram superadas e consideradas naturais na adaptação ao novo ambiente. $^{3}$

Algumas dificuldades e desafios enfrentados pelos estudantes brasileiros no exterior foram: o idioma, afetando a comunicação, dificuldade na aprendizagem, as diferentes terminologias e rotinas nas práticas dos cursos de enfermagem. ${ }^{5}$ A imersão linguística e cultural permite 0 aprimoramento e fluência, especificamente na língua 


\section{JOURNALOF \\ NURSING \\ ANO HEALTH}

ISSN 2236 - 1987

entrevistas em francês ou inglês, provas e validação do diploma. Precisam cursar algumas disciplinas em Universidades locais antes de poderem trabalhar, por não serem contempladas no currículo da graduação no Brasil. Em média, os processos de tramitação dos documentos duram de três a cinco anos e observa-se que quanto mais elevada a idade do candidato há menos chance de ser aprovada a documentação no consulado Canadense.

\section{CONSIDERAÇÕES FINAIS}

0 intercambio, foi uma oportunidade para agregar valores, aprimorar o currículo, ampliar o conhecimento e a dedicação aos estudos na área da enfermagem, aprofundar conhecimentos teóricos e científicos sobre a fisiopatologia das cardiopatias e nos cuidados a pacientes críticos, além de conhecer diferentes linhas de pesquisas e teorias voltadas para a área da saúde. Proporcionou crescimento pessoal e profissional, vivência cultural, valores, vínculos e experiências significativas no contexto da formação em saúde, bem como do pesquisador e docente na área da saúde.

Nesse sentido, 0 pensamento crítico-reflexivo foi estimulado e desenvolvimento a partir de situações diversas, de forma a buscar soluções diante de problemas e dificuldades com os recursos disponíveis.

Aspectos limitantes dizem respeito à falta de comunicação eficiente do idioma local, o francês. Contudo, através desse relato esperase contribuir para que estudantes se preparem antecipadamente para 
realizar um intercâmbio. Dessa forma, recomenda-se previamente analisar a linha de pesquisa do coorientador estrangeiro, a cultura local, reservar moradia, pesquisar valores e a moeda utilizada, levar os documentos atualizados, pesquisar o clima e os tipos de roupas que se deve levar ou se preparar para aquisição no local.

\section{REFERÊNCIAS}

1 Morosini MC. Internacionalização na produção de conhecimento em IES brasileiras: cooperação internacional tradicional e cooperação internacional horizontal. Educ. rev. [Internet]. 2011[acesso em 2018 out 18];27(1):93112.

Disponível em: http://www.scielo.br/pdf/edur/v27n 1/v27n1a05.pdf

2 Fassarella CS, Silva LD, Figueiredo MCB. PhD courses in nursing in a cotutorial international regime: a chance to be experimented. Rev. enferm. UERJ. [Internet]. 2013[cited 2018 Oct. 18];21(5):682-6. Available from: http: / /www.epublicacoes.uerj.br/index.php/enfer magemuerj/article/view/10055/9033

3 Oliveira MG, Pagliuca LMF. Programa de mobilidade acadêmica internacional em enfermagem: relato de experiência. Rev. gaúch. enferm. [Internet]. 2012[acesso em 2018 out 18];33(1):195-8. Disponível em: http://www.scielo.br/pdf/rgenf/v33n 1/a26v33n1.pdf

4 Dalmolin IS, Pereira ER, Silva RMCRA, Gouveia MJB, Sardinheiro JJ. Intercâmbio acadêmico cultural internacional: uma experiência de crescimento pessoal e científico. Rev. bras. enferm. [Internet]. 2013[acesso em 2018 out 18];66(3):442-7.
Disponível http://www.scielo.br/pdf/reben/v66 n3/a21v66n3.pdf

5 Carvalho Jl, Backes DS, Lomba MLLF, Colomé JS. Intercâmbio académico internacional: uma oportunidade para a formação do futuro enfermeiro. Revista de enfermagem referência [Internet]. 2016[acesso em 2018 out 18];5(10):59-67. Disponível em: http://www.scielo.mec.pt/pdf/ref/vs erIVn10/serIVn10a07.pdf

6 Faria LR, Alves CA. On caring: preliminaries of a comparative study of primary health care in Brazil/Canada. Saúde Soc. [Internet]. 2015[cited 2018 Oct 18];24(1):1-14. Available from: http: //www.scielo.br/pdf/sausoc/v24 n1/en_0104-1290-sausoc-24-10072.pdf

7 Oliveira RAA, Zago MMF, Thorne SE. Interaction between professionals and cancer survivors in the context of brazilian and canadian care. Rev. latinoam. enferm. (Online). [Internet]. 2017[cited 2018 Oct. 18];25:e2972. Available from: http://www.scielo.br/pdf/rlae/v25/0 104-1169-rlae-25-e2972.pdf

8 Melo M. Relato de experiência: ciência sem fronteiras na Université Du Québec À Trois-Rivières - Canadá. Revista uniplac [Internet]. 2017[acesso em 2019 jan 07];5(1). Disponível em: http://revista.uniplac.net/ojs/index. $\mathrm{php} /$ uniplac/article/view/2625

9 Ratković S, Kovačević D, Brewer CA, Ellis C, Ahmed N, Baptiste-Brady J. Supporting refugee students in Canada: building on what we have learned in the past 20 years [Internet]. 2017[cited 2018 Oct. 18]. Available from: 


\section{NURSING \\ AND HEALTH}

http: //citiesofmigration.ca/wpcontent/uploads/2018/04/SupportingRefugee-Students-in-Canada-FullResearch-Report-1.pdf

10 Ferrari RFR, Rodrigues DMMR, Baldissera VDA, Pelloso SM, Carreira L. Aplicabilidade da teoria de Virginia Henderson para fundamentação na enfermagem: fragilidades e potencialidades. Arq. ciências saúde UNIPAR. [Internet]. 2014[acesso em 2018 out 18];18(1):51-6. Disponível em:

http: / / revistas.unipar.br/index.php/s aude/article/view/5158/2984

11 Makuch DMV, Zagonel, IPS. Abordagem pedagógica na implementação de programas curriculares na formação do enfermeiro. Esc. Anna Nery Rev. Enferm. [Internet]. 2017[acesso em 2019 jan 07];21(4):e20170025. Disponível em: http://www.scielo.br/pdf/ean/v21n4 /pt_1414-8145-ean-2177-9465-EAN2017-0025.pdf

12 Silva WLAV, Barros ATL, Santos RD, Silva LA, Miranda LN. Cirurgias cardíacas: assistência de enfermagem a portadores de cardiopatia no período perioperatório. Cadernos de graduação ciências biológicas e da saúde unit Alagoas [Internet]. 2017[acesso em 2019 jan 07];4(2):323-36. Disponível em:

https: / / periodicos.set.edu.br/index.p $\mathrm{hp} /$ fitsbiosaude/article/view/4565/26 25 